\title{
CALCULATIONS OF ION TRAJECTORIES AT MAGNETOPLASMA SEPARATION AND EXPERIMENTS WITH POLYATOMIC GASES
}

\author{
V. B. Yuferov*, S. V. Shariy, T. I. Tkachova, V. V. Katrechko, \\ A. S. Svichkar, V. O. Ilichova, M. O. Shvets, E. V. Mufel \\ National Science Center "Kharkov Institute of Physics and Technology", 1 Akademicheskaya Str., Kharkov, \\ * corresponding author: v.yuferov@kipt.kharkov.ua
} 61108, Ukraine

\begin{abstract}
Calculated trajectories of ions with different masses, indicating the possibility of a mixture separation, are obtained. Comparative experiments for plasma of monatomic and polyatomic gases (Ar, $\mathrm{N}_{2}, \mathrm{CO}_{2}$ ), upon combination of pulsed discharge with a stationary one with incandescent cathode, are carried out. The oscillograms of discharge current and voltage at low emission currents and a constant energy input show that energy is spent on other processes different from ionization. With an increase of emission current, the nonlinear character of the discharge current and voltage, which may be indicative of the role of dissociation and vibrational levels in energy consumption, is observed. In addition, there is connection between the number of atoms in molecule and the values of maximum discharge current and the pressure of injected gas.
\end{abstract}

KEYWORDS: spent nuclear fuel; plasma reprocessing; uranium; fission products.

\section{INTRODUCTION}

At present, the recycling of nuclear fuel (NF), i.e., its reuse, is implemented by using PUREX-process. However, it leads to an increase of liquid radioactive waste (RW) volume, while the alternative methods of a physical reprocessing, in particular plasma ones, do not require chemical reagents, but use only electrical power. Currently, researches on plasma reprocessing of spent nuclear fuel (SNF) are carried out in the United States, Russia and Ukraine [1-11]. In the NSC "KIPT", the magnetoplasma reprocessing of the SNF is offered, which includes three stages (heating, ionization and magnetoplasma separation in rotating plasma), where fission products (FP) are consistently separated from nuclear fuel (NF) [12] without chemical pretreatment of irradiated fuel, suggested in [1, 6. To reduce the number of components during transfer of the SNF into plasma and thus energy costs, the stage of the thermal heating is required, which allows removing up to $75 \%$ of the $\mathrm{FP}$, whereupon the mixture with the NF contains only zirconium and lanthanides oxides. Currently, experiments are carried out with multicomponent gas molecular plasma. For a more complete approach to the SNF in $[12,13]$, the choice of simulation media with elements that are a part of the SNF, but are not radioactive, was offered. Thus the simulation of the SNF separation, consisting of $\sim 90 \%$ of the uranium dioxide and $\sim 10 \%$ of the FP (according to the number of particles), should be carried out in a multicomponent molecular medium. To simulate the SNF plasma, the process includes a creation of plasma from multicomponent mixture of metals and oxides, i.e., elements and compounds with different values of dissociation and ionization energies and recycling coefficients.

\section{Calculations of MOleCUlar iONS TRAJECTORIES IN SNF PLASMA ROTATED IN EXH FIELDS}

At the SNF heating 12 the amount of the FP can be significantly reduced due to removal of the FP with dissociation energy $\left(\varepsilon_{i}\right)$ less than their own ionization energy $\left(\phi_{i}\right)$ and dissociation energy of uranium oxide $(\varepsilon): \varepsilon_{i}<\phi_{i}<\varepsilon$. Figure 1 shows the dissociation energies and ionization potentials of the FP oxides before and after heating.

As can be seen from Figure 1b, after heating (up to $2500{ }^{\circ} \mathrm{C}$ ) and thermal desorption, the SNF contains oxides of actinides, lanthanides, and of zirconium, with $\varepsilon_{i} \geq \varepsilon>\phi_{i}$, i.e., during subsequent ionization, the SNF plasma will contain molecular ions of actinides oxides $\left(\mathrm{UO}_{2}{ }^{+}, \mathrm{PuO}_{2}{ }^{+}, \mathrm{UO}^{+}, \mathrm{PuO}^{+}\right)$and molecular ions of the FP, such as: $\mathrm{ZrO}^{+}, \mathrm{CeO}_{2}{ }^{+}, \mathrm{La}_{2} \mathrm{O}_{3}{ }^{+}$, $\mathrm{Nd}_{2} \mathrm{O}_{3}{ }^{+}$and others.

Molecular ions of the FP and the NF can be separated at the magnetoplasma separation stage [12]. The separation of ions occurs due to their different trajectories in collisionless plasma rotated in crossed electric and magnetic fields. The mathematical model used in this paper is described in 13 . Note that this mathematical model is based on the balance of the forces acting on the charged particles and describes their motion in a plasma rotating in the ExH fields. It is also used in [1,4] and allows to estimate the parameters of the separation process in a collisionless plasma. 

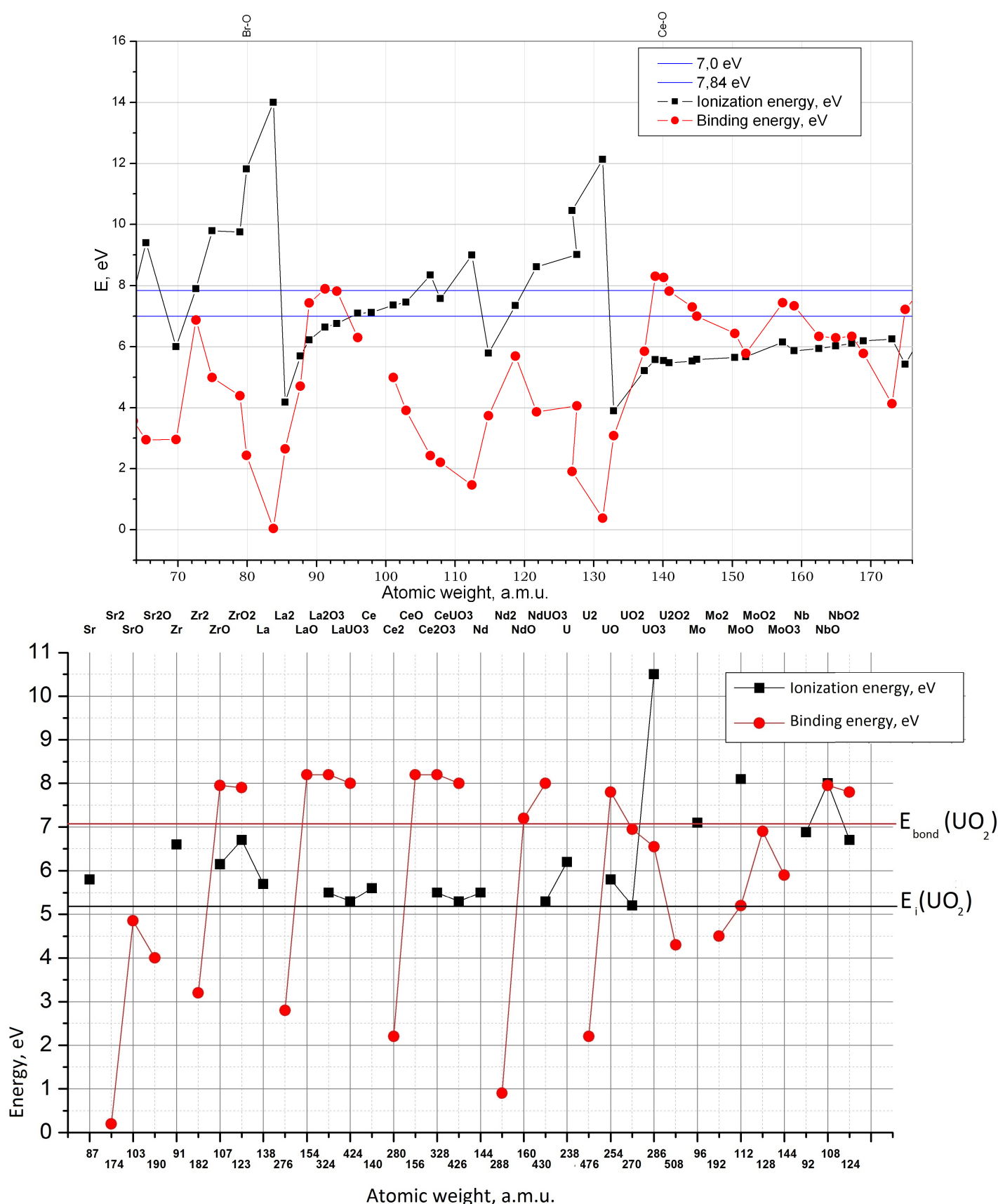

Figure 1. Dissociation energies of the FP oxides and potentials of their ionization: (a) before and (b) after the thermal heating stage.

The induction of magnetic field has two components: $B_{r}$ and $B_{z}$. The axial component $B_{z}$ is shown in Figure 2 (curve 5). The $B_{r}$ is associated with $B_{z}$ by ratio $\operatorname{div} B=0$. The electric field intensity has a radial component $E r \neq 0$. Fig. 2, a shows trajectories of ions with mass numbers 106, 160, 270 and 324 (curves 1, 2, 3 and 4, respectively). The mass numbers 106, 160 correspond to ions of the FP oxides, which remain in the system after heating and ionization. Mass 270 a.m.u. corresponds to a molecular mass of uranium dioxide $\left(\mathrm{UO}_{2}\right)$, i.e., NF. Figure 2 a shows that the NF can be separated from the FP in that system. The horizontal line (6) corresponds to the prospective radius of chamber and it can be seen that
$\mathrm{UO}_{2}{ }^{+}$ions reach the chamber walls where the collector for the NF is located, while the FPs move along the trajectories, with a smaller Larmor radius, towards the chamber end to the collector for the FPs. However, the difficulty is in formation of the SNF of molecular ions of lanthanide oxides such as $\mathrm{La}_{2} \mathrm{O}_{3}{ }^{+}$, with mass greater than mass of uranium dioxide. Figure 2 a shows that the trajectory of the $\mathrm{La}_{2} \mathrm{O}_{3}{ }^{+}$ion (324 a.m.u.) crosses the prospective radius of the chamber at the same point as the $\mathrm{UO}_{2}{ }^{+}$fuel ion, i.e., collector for the $\mathrm{NF}$ will also collect oxides such as $\mathrm{La}_{2} \mathrm{O}_{3}$.

One of the possible solutions of this problem is to add a variable component to the constant radial 

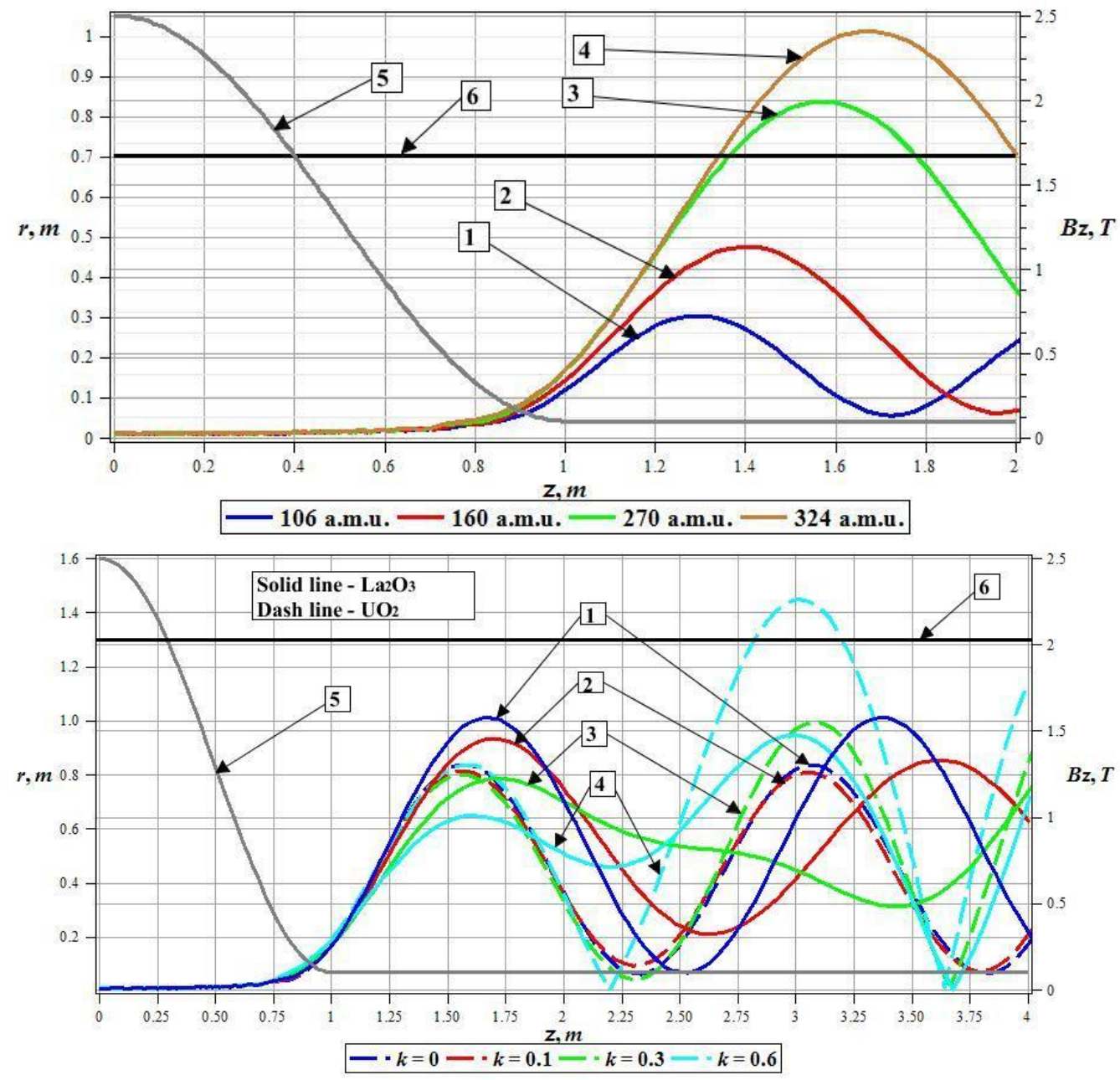

Figure 2. (a) Trajectories of ions with different masses at $\alpha=45^{\circ}, r_{0}=0.01 \mathrm{~m}, W=5 \mathrm{eV}$. (b) Trajectories of $\mathrm{UO}_{2}{ }^{+}$ ions (dash lines) and $\mathrm{La}_{2} \mathrm{O}_{3}{ }^{+}$ions (solid lines) at $\omega=\frac{1}{2} \omega_{\text {ci }}\left(\mathrm{UO}_{2}\right)$.

electric field [5]:

$$
E_{\mathrm{r}}=E_{0}+E_{\mathrm{add}}, \quad E_{\mathrm{add}}=k E_{0} \sin \omega t .
$$

The value $B_{0}$ corresponds to a maximum magnetic field. The value $E_{0}$ was considered to be $400 \mathrm{~V} / \mathrm{m}$. In the calculations, $\mathrm{k}$ is equal to $0,0.1,0.3$ and 0.6 (curves 1, 2, 3 and 4, respectively in Fig. 2, b). The frequency $\omega$ is related to the ion cyclotron frequency in the region of uniform magnetic field, where $B=B_{0} / 25$ :

$$
\omega_{\mathrm{ci}}=\frac{q}{m} \frac{B_{0}}{25}
$$

Calculations show that in the case of $\omega=\frac{1}{2} \omega_{\text {ci }}\left(\mathrm{UO}_{2}\right)$ (Figure $2 \mathrm{p}$ ), the trajectory of the $\mathrm{UO}_{2}{ }^{+}$ion radially exceeds the trajectory of the $\mathrm{La}_{2} \mathrm{O}_{3}{ }^{+}$ion at $k=0.6$. This means that the addition of variable component leads to the fact that the collector for the NF will collect only particles with mass 270 a.m.u., and the FPs, as the $\mathrm{La}_{2} \mathrm{O}_{3}{ }^{+}$ions, will move towards the chamber end to a corresponding collector. In Fig. 2, b horizontal line (6) corresponds to the prospective radius of the chamber.

\section{EXPERIMENTS WITH PLASMA OF MONATOMIC AND POLYATOMIC GASES}

In [8], it is pointed out that the SNF reprocessing is accompanied with energy costs of the uranium ion at the level of $500-1000 \mathrm{eV} /$ atom. However, when creating the SNF molecular plasma, it is necessary to consider its multicomponent nature. Therefore, the choice of the simulation media (SM) mixture of monatomic and polyatomic gases was selected. Initially, the qualitative assessment of plasma characteristics in experiments with individual gases, such as $\mathrm{Ar}, \mathrm{N}_{2}, \mathrm{CO}_{2}$, was carried out.

Comparative experiments were carried out in the cylindrical chamber with a profiled magnetic field. In the stationary mode, when working with nitrogen plasma, the voltage and current at the discharge gap of plasma source with incandescent cathode were $\sim 50 \mathrm{~V}, 10 \mathrm{~A}$. However, as optical measurements have shown, only molecular plasma of $\mathrm{N}_{2}{ }^{+}$ions was obtained. From the energy point of view, the $\mathrm{N}^{+}$ions with lower ionization energy $\left(\phi_{i}=14.54 \mathrm{eV}\right)$ than the $\mathrm{N}_{2}{ }^{+}\left(\phi_{i}=15.5 \mathrm{eV}\right)$ should have higher density. For 


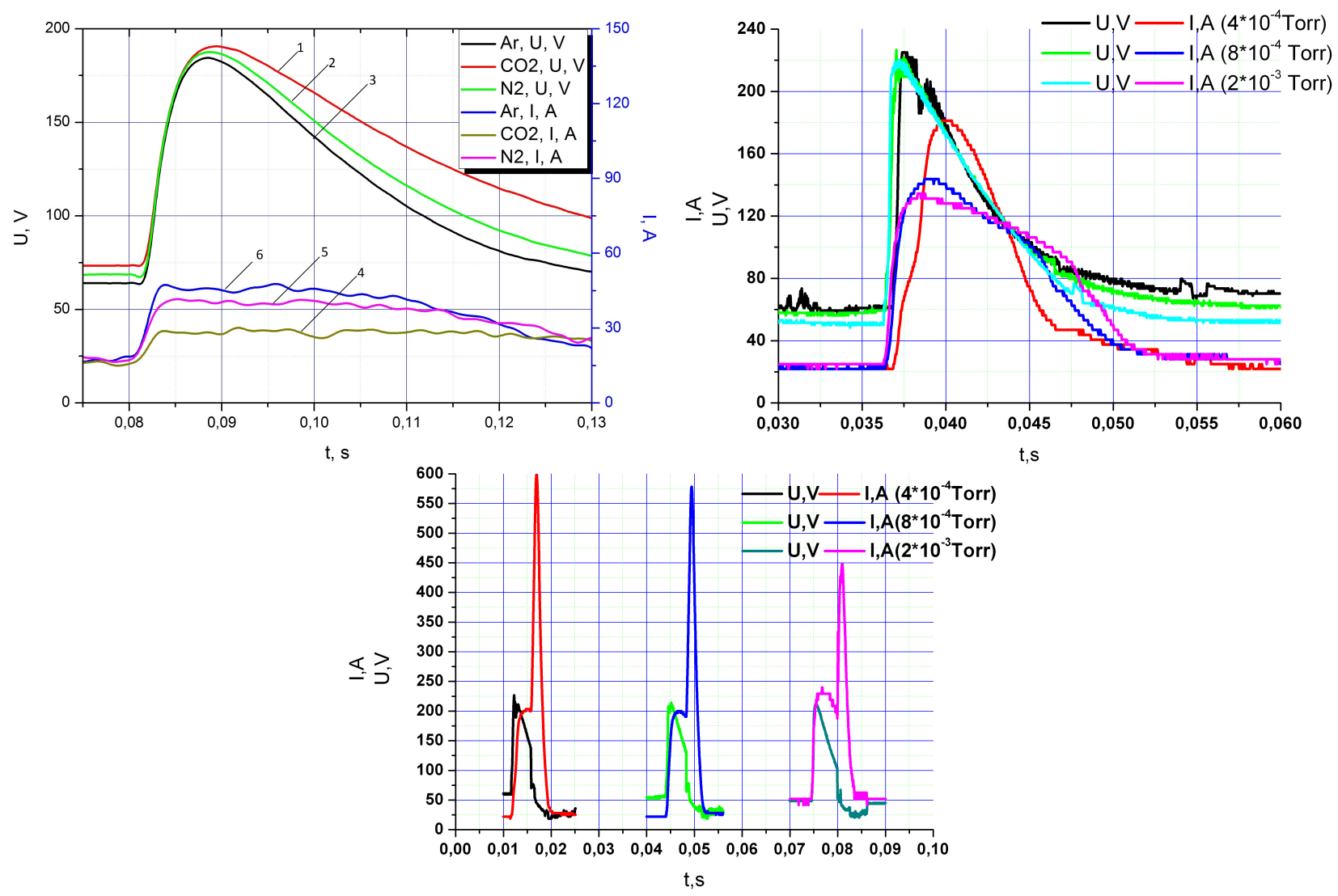

Figure 3. Current-voltage characteristics of discharge in (a) $\mathrm{Ar}, \mathrm{N}_{2}, \mathrm{CO}_{2}$, (b) Ar and (c) $\mathrm{CO}_{2}$ versus time at parameters $H=\alpha I(\alpha=1.8)$, current in magnetic coils $125 \mathrm{~A}$.

collisionless plasma in limited size systems, the reaction $\mathrm{N}_{2}+\mathrm{e}=2 \mathrm{~N}+\mathrm{e}$ is more probable, because of the dissociation energy $\varepsilon_{i}<\phi_{i}$. Thus, neutral atoms, not held by a magnetic field, move to the chamber wall where they recombine into molecule $\mathrm{N}_{2}$ and are returned to the discharge volume. Consequently, for formation of the $\mathrm{N}^{+}$ions, it is necessary to increase the value $T_{\mathrm{e}}$, in order to proceed to processes of dissociative ionization: $\mathrm{N}_{2}+\mathrm{e}=\mathrm{N}+\mathrm{N}^{+}+2$ e, i.e., increase the energy input into the discharge. It is expected that a similar situation will be in multicomponent plasma of the SNF.

Increasing of the energy input in the stationary mode is not possible due to the thermal loads on the elements of plasma source and power supply limitations. Therefore, additional pulse source was used (battery capacity $6.6 \mathrm{mF}$, voltage up to $300 \mathrm{~V}$ with the inductance, which determines the pulse duration [12]). However, when the pulse is superimposed on plasma discharge, the situation changes. As can be seen from Figure $3 \mathrm{a}$, at the same initial conditions, pulse amplitudes and durations for $\mathrm{Ar}, \mathrm{N}_{2}, \mathrm{CO}_{2}$ are different.

Current and voltage oscillograms (Figure $3 \mathrm{k}$ ) at low emission currents of about $1 \mathrm{~A}$ and constant power input show that maximum current in time corresponds to a one third of voltage, further increase of voltage does not lead to the increase of charged particles number and energy is spent on other processes. Reduction of discharge currents for different gases with the same geometry of discharge chambers indicates reduction of density and temperature of electrons and ions. This may be due to the fact that energy is directed into degrees of freedom, i.e., into excitation of rotational and vibrational levels of the molecules. So, the energy expenditure of the ion in the $\mathrm{CO}_{2}$ plasma is higher than of the one in the $\mathrm{N}_{2}$ plasma, and the energy expenditure of the ion in the $\mathrm{N}_{2}$ plasma is higher than of the one in the Ar plasma, respectively.

With the increase of emission currents of the incandescent cathode up to $3 \mathrm{~A}$, discharge currents for $\mathrm{Ar}$ (Figure 3b) and $\mathrm{CO}_{2}$ (Figure 3r) increase sharply, i.e., in the case of $\mathrm{CO}_{2}$, energy is spent on dissociation and ionization with increasing number of particles, and in the case of Ar, number of particles is constant. Due to the increase in number of particles in the case of $\mathrm{CO}_{2}$, discharge current is increased and pulse duration is reduced.

The increased pressure in the experiments with $\mathrm{CO}_{2}$ leads to a decrease in amplitude discharge current, which may indicate the reduction of ionization in favor of the dissociation processes.

\section{TWO-STAGE Plasma SOURCE}

The transition to gas-metal mixtures, similar to the SNF composition by physical and chemical properties, will allow working out the principles of magnetoplasma reprocessing of the SNF [11. It should be noted that 


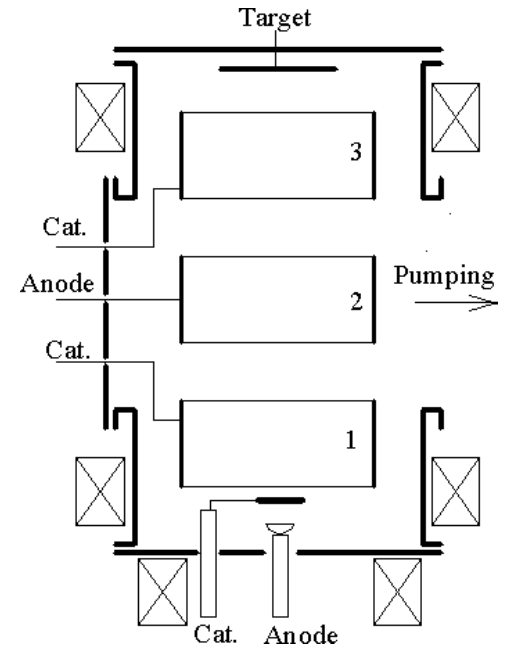

Figure 4. The scheme of the two-stage plasma source.

a power reactor with a capacity of $1 \mathrm{GW}$ annually accumulates $5-10$ tons of the SNF $(1 \mathrm{~kg} / \mathrm{h})$. Thus, the special problem is the creation of effective plasma sources with great productivity.

For this purpose, the development of two-stage plasma source is carried out. The project of the plasma source and the description of its components is presented in [14. In the first stage of the research, copper was selected as the working substance. The use of copper in a large number of different experiments will facilitate a comparative analysis of the parameters of the source with the previously obtained data.

The two-stage plasma source is schematically presented in Figure 4 the first stage - (cathode-anode) arc discharge with incandescent cathode in magnetic field; the second stage - reflective discharge $(1,2,3$ - electrodes). The cathode and electrodes 1, 3 have zero potential. The anode and electrode 2 were under voltage up to $+150 \mathrm{~V}$ vs. zero potential.

Figure 5 shows the dependences of discharge currents from the discharge voltage on liquid anode $I_{\text {dis }}$ and Penning discharge electrodes $I_{1}, I_{2}, I_{3}$.

At the initial stage, the discharge is ignited and burns puffed gas (air). The pressure in the chamber is at the level of $5 \cdot 10^{-4}$ Torr. The currents $I_{1}, I_{2}$, $I_{3}$ increase almost linearly. Further, with increase of current to the tungsten crucible-anode, the working metal in it melts and the vapors enter into the discharge region. There is a quite sharp change of current on electrodes and a change of the discharge glow. Characteristically, the electrodes' currents $I_{1}$, $I_{2}$ increase, and $I_{3}$ decrease. After the start of the copper evaporation, the puffed gas in discharge chamber was stopped and discharge occurs in metal vapor, the pressure in vacuum chamber was set at the level of $2 \cdot 10^{-5}$ Torr. This is due to the getter pumping residual gas by atoms and ions of the evaporated metal. Figure $5 \mathrm{~b}$ shows radial distribution of floating potential in the region between the electrodes 2 and 3 . The measurements were carried out using single Langmuir probe. Measurements of floating potential in regard to zero potential of the vacuum chamber walls were carried out. The characteristic feature is the tubular distribution of plasma potential with a sharp rise in the axial region.

At a power input of $3 \mathrm{~kW}$ in the experiments, the amount of evaporated copper of up to $\sim 100 \mathrm{~g} / \mathrm{h}$ was obtained. In the future, we plan to carry out a forming of plasma of the metals and metal oxides, which are a simulation material of the SNF.

\section{SUMmary}

(1.) Multistage magnetoplasma reprocessing of the SNF assumes that after heat treatment, the SNF composition will contain, mainly, compounds with dissociation energy greater than uranium dioxide one. In the plasma ionization stage, it is possible to partially separate out the zirconium oxides, as its ionization potential is greater than uranium dioxide one, in contrast to lanthanides and their oxides. Further purification of the SNF from the FPs is possible in rotating plasma, where ions such as $\mathrm{La}_{2} \mathrm{O}_{3}{ }^{+}$ with mass numbers $\sim 320$ can be separated from the NF oxides by applying a variable component $E_{\text {add }}$ with frequency $\omega_{\text {ci }} / 2$ on the constant electric field.

(2.) The experiments with plasma of molecular gases at low emission currents showed that increase of atom number in molecules of working gas leads to the decrease of discharge current. It follows that energy is spent not only on the ionization of working gas, but on the excitation of vibrational and rotational levels as well. The increase of electron emission from incandescent cathode leads to a sharp increase of discharge current. In the case of $\mathrm{CO}_{2}$, unlike argon, the dependence of discharge current on time has a two-stage nature, which is provided by the dissociation of molecules, increase of the particle number and their subsequent ionization.

(3.) In the studied two-stage plasma source, the rate of copper evaporation reached up to $\sim 100 \mathrm{~g} / \mathrm{h}$ at a power input of $3 \mathrm{~kW}$.

\section{REFERENCES}

[1] Litvak A. Archimedes Plasma Mass Filter / Litvak A., Agnew S., Anderegg F,et al // 30th EPS Conference on Contr. Fusion and Plasma Phys. - St. Petersburg (Russia). 2003. - Vol. 27A, O-1.6A.

[2] Winslow, D. L.; Agnew, S. F.; Anderegg, F.; Cluggish, B. P.; Freeman, R. L.; Gilleland, J.; Hilsabeck, T. J.; Isler, R. C.; Lee, W. D.; Litvak, A.; Miller, R. L.; Ohkawa, T.; Putvinski, S.; Umstadter, K. R.; Zhang, J. Plasma Generation and Mass Separation in the Archimedes Demonstration Unit // 2004 APS.DPPEP1087W.

[3] Winslow, D. L. Mass Separation of Nuclear Waste Surrogates in the Archimedes Demonstration Unit // American Physical Society, 47th Annual DPP Meeting, October 24-28, 2005, abstract \#KP1.074. 

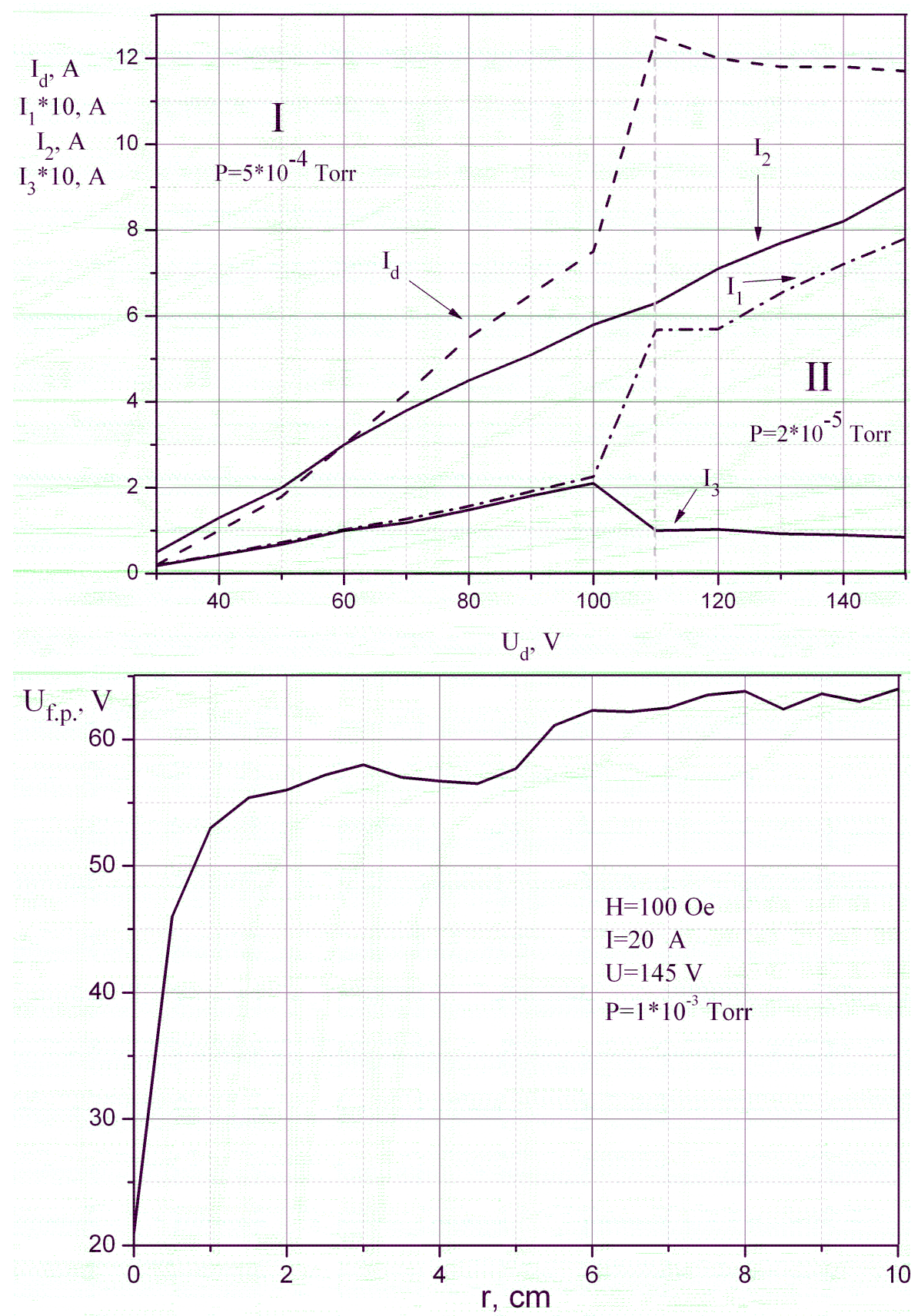

FigURE 5. (a) $I_{\mathrm{d}}$ - discharge current with incandescent cathode (the first stage of plasma source); $I_{1}, I_{2}, I_{3}-$ currents on electrodes of reflective discharge (the second stage of plasma source). (b) Floating potential versus the radial distance from the axis of the vacuum chamber. The diameter of the reflective discharge electrodes is $10 \mathrm{~cm}$.

[4] Freeman R. et al., Archimedes Plasma Mass Filter, DOI:10.1063/1.1638067

[5] Ohkawa T and Miller R. L., Band gap ion mass filter, DOI:10.1063/1.1523930

[6] R. Gueroult and N.J. Fisch. Plasma mass filtering for actinides lanthanides separation // Princeton Plasma Phys. Lab. PPPL-4944/Oct. 2013

[7] Fetterman J. and Fisch N. J., Wave-driven countercurrent plasma centrifuge DOI:10.1088/0963-0252/18/4/045003

[8] V.A. Zhil'tsov, V.M. Kulygin, N.N. Semashko et.al. Plasma separation of the elements applied to nuclear materials handling // Atomic Energy, 2006. Vol. 101, No. 4, pp.302-306.

[9] Timofeev A.V., On the theory of plasma processing of spent nuclear fuel, DOI:10.3367/UFNe.0184.201410g.1101

[10] A.M. Yegorov, V .B. Yuferov, S.V. Shariy, V.A. Seroshtanov, O.S. Druy, V.V. Yegorenkov, S.N .Khizhnyak, D.V. Vinnikov // Preliminary Study of the Demo Plasma Separator // Problems of Atomic Science and Technology, 2009. No. 1(59), pp.122-124.

[11] V.B. Yuferov, A.M. Yegorov, V.O. Ilichova, S.V. Shariy, K.I. Zhivankov Plasma Separation of Spent Nuclear Fuel - One of Possible Ways to Solve a 
Problem of Closed Fuel Cycle // Problems of Atomic Science and Technology, 2013. No. 2(84), pp. 148-151.

[12] V.B. Yuferov, V.V. Katrechko, A.S. Svichkar, S.V. Shariy, T.I. Tkachova, E.V. Mufel, V.O. Ilichova, A.Yu. Pakhomov Problems of Impuruties Deducing from Multicomponent Media at Thermal Heating, Ionization and Rotation of Plasma in Crossed Fields // Problems of Atomic Science and Technology, 2016. No. 1(101), pp.124-130.
[13] V.B. Yuferov, V.V.Katrechko, T.I,Tkachova, S.V. Shariy,A.S.Svichkar,E.V.Mufel, V.O.Ilichova, M.O.Shvets. Some Questions of SNF Reprocessing at the Stages of Ionization and Magnetoplasma Separation in Crossed Fields // Problems of Atomic Science and Technology, 2015. No. 4(98), pp.345-349.

[14] V.B. Yuferov, S.V. Shariy, M.O. Shvets, A.N. Ozerov. Gas-metal plasma source project for the separation technology // Problems of Atomic Science and Technology. 2014. No. 5(93), pp.184-187. 